\title{
Nonselective carotid artery ultrasound screening in patients undergoing coronary artery bypass grafting: Is it necessary?
}

\author{
Khalil Masabni, MD, ${ }^{a}$ Joseph F. Sabik III, MD, ${ }^{a}$ Sajjad Raza, MD, ${ }^{\mathrm{a}}$ Theresa Carnes, MPH, ${ }^{\mathrm{b}}$ \\ Hemantha Koduri, MD, ${ }^{\mathrm{c}}$ Jay J. Idrees, MD, ${ }^{\mathrm{a}}$ Jocelyn Beach, MD, ${ }^{\mathrm{d}}$ Haris Riaz, MD, \\ Mehdi H. Shishehbor, DO, MPH, PhD, ${ }^{f}$ Heather L. Gornik, MD, ${ }^{c}$ and Eugene H. Blackstone, MD ${ }^{\mathrm{a}, \mathrm{b}}$
}

\begin{abstract}
Objectives: To determine whether nonselective preoperative carotid artery ultrasound screening alters management of patients scheduled for coronary artery bypass grafting (CABG), and whether such screening affects neurologic outcomes.

Methods: From March 2011 to September 2013, preoperative carotid artery ultrasound screening was performed on 1236 of 1382 patients $(89 \%)$ scheduled to undergo CABG. Carotid artery stenosis (CAS) was classified as none or mild (any type 0\%-59\% stenosis), moderate (unilateral 60\%-79\% stenosis), or severe (bilateral 60\%-79\% stenosis or unilateral 80\%-100\% stenosis).
\end{abstract}

Results: A total of 1069 (86\%) had <moderate; 90 (7.3\%) had moderate; and 77 $(6.2 \%)$ had severe CAS. Of those with moderate CAS, $4(4.4 \%)$ had preoperative confirmatory testing, and $1(1.1 \%)$ underwent combined CABG + carotid endarterectomy (CEA); 11 (12\%) had off-pump surgery. Of those with severe CAS, $18(23 \%)$ had confirmatory testing, and $18(23 \%)$ underwent combined CABG + CEA; $6(7.8 \%)$ had off-pump surgery. Stroke occurred in 14 of 1069 $(1.3 \%)$ patients with $<$ moderate CAS; 2 of $90(2.2 \%)$ of those with moderate CAS; and 2 of $77(2.6 \%)$ of those with severe CAS $(P=.3)$. In patients with $\geq$ moderate CAS, 1 of $19(5.3 \%)$ undergoing CABG + CEA and 3 of 148 $(2.0 \%)$ undergoing $\mathrm{CABG}$ alone experienced stroke $(P=.4)$. In patients with moderate CAS, stroke occurred in 1 of $11(9.1 \%)$ off-pump and 1 of $79(1.3 \%)$ on-pump patients $(P=.2)$. In patients with severe CAS, stroke occurred in 1 of $6(17 \%)$ off-pump and 1 of $71(1.4 \%)$ on-pump patients $(P=.15)$.

Conclusions: Routine preoperative carotid artery evaluation altered the management of a minority of patients undergoing $\mathrm{CABG}$; this did not translate into perioperative stroke risk. Hence, a more targeted approach for preoperative carotid artery evaluation should be adopted. (J Thorac Cardiovasc Surg 2016;151:402-9)

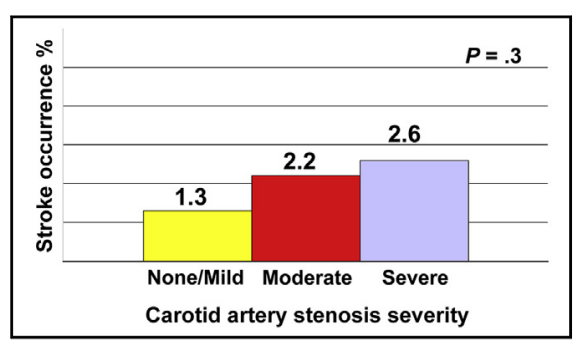

Stroke occurrence by severity of carotid artery stenosis in patients undergoing coronary artery bypass grafting.

\section{Central Message}

Routine, as opposed to selective, preoperative carotid artery ultrasound screening before coronary artery bypass grafting is of questionable value.

\section{Perspective}

Routine preoperative carotid artery evaluation altered the management of a minority of patients undergoing $\mathrm{CABG}$. These changes in management did not decrease the prevalence of perioperative stroke. Hence, in patients undergoing $\mathrm{CABG}$, a more targeted approach for preoperative carotid artery evaluation should be adopted.

See Editorial Commentary page 410.
Stroke is a devastating complication of coronary artery bypass grafting $(\mathrm{CABG})^{1}$ that is associated with substantial morbidity, mortality, and costs for both patients and healthcare systems. With the expectation of reducing the

\footnotetext{
From the Departments of ${ }^{a}$ Thoracic and Cardiovascular Surgery, ${ }^{c}$ Vascular Medicine, ${ }^{\mathrm{d}}$ Vascular Surgery, and ${ }^{\mathrm{f} C a r d i o v a s c u l a r}$ Medicine, Heart and Vascular Institute; ${ }^{\mathrm{b}}$ Department of Quantitative Health Sciences, Research Institute; and ${ }^{\mathrm{e}}$ Department of Internal Medicine, Medicine Institute, Cleveland Clinic, Cleveland, Ohio.

This study was supported in part by the Sheikh Hamdan bin Rashid Al Maktoum Distinguished Chair in Thoracic and Cardiovascular Surgery, held by J.F.S.; the Kenneth Gee and Paula Shaw, PhD, Chair in Heart Research, held by E.H.B.; and the Gus P. Karos Registry Fund. The individuals for whom these funding sources are named played no role in the collection of data or analysis and interpretation of the data, and had no right to approve or disapprove publication of the finished article.
}

occurrence of perioperative stroke, some groups routinely screen patients before CABG to identify carotid artery disease that they believe requires revascularization before or during $\mathrm{CABG}^{2-7}$ In 2011, we implemented a quality 


\section{Abbreviations and Acronyms \\ $\mathrm{CABG}=$ coronary artery bypass grafting \\ $\mathrm{CEA}=$ carotid endarterectomy}

\section{Scanning this QR code will} take you to the article title page. To view the AATS 2015 Webcast, see the URL at the end of the article.

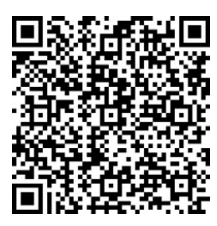

improvement protocol to reduce the occurrence of strokes, which included universal carotid artery screening. The purposes of this study were to determine whether this quality improvement protocol (1) altered the management of patients scheduled to undergo isolated CABG, and (2) affected neurologic outcomes.

\section{METHODS}

\section{Patients}

From March 2011 to September 2013, nonselective carotid artery ultrasound screening was performed prospectively on 1236 of $1382(89 \%)$ patients scheduled to undergo isolated $\mathrm{CABG}$ at Cleveland Clinic. Preoperative, operative, and postoperative variables were retrieved from the Cardiovascular Information Registry, a database populated concurrently with patient care information. Medical records of all patients who were recorded as having carotid artery stenosis, identified on screening ultrasound, were reviewed to identify any change in perioperative management that occurred on that basis. Medical and imaging records of patients experiencing an adverse neurologic event were reviewed. The average age of screened patients was $65 \pm 10$ years, and $75 \%$ were male, with no statistically significant differences between those who were, versus were not, screened (Table 1). Data used in this study were approved for use in this quality improvement project by the Cleveland Clinic Institutional Review Board.

\section{Carotid Artery Screening}

Most of the carotid duplex ultrasound examinations (92\%) were performed in the Non-Invasive Vascular Laboratory of Cleveland Clinic, a facility accredited by the Intersocietal Accreditation Commission (www.intersocietal.org) in extracranial cerebrovascular testing. Studies were performed by registered vascular technologists using a standardized scanning protocol, and interpreted by vascular medicine specialists and vascular surgeons certified in vascular interpretation. Duplex ultrasound examination findings and results were entered into an electronic database at the time of interpretation. The median interval from screening ultrasound to CABG was 4 days, with $90 \%$ performed within 16 days. Each internal carotid artery was graded and categorized according to the severity of stenosis, based on standardized laboratory diagnostic criteria, ${ }^{8}$ including internal carotid artery peak systolic and end-diastolic velocities and presence of atherosclerotic plaque.

Results of the carotid artery ultrasound were classified as follows: no stenosis or mild stenosis (any type $<60 \%$, including categories $0 \%-19 \%, 20 \%-39 \%$, and $40 \%-59 \%$ ); moderate stenosis (unilateral, $60 \%-79 \%$ stenosis of either internal carotid artery); or severe stenosis (bilateral 60\%-79\% stenosis; unilateral or bilateral 80\%-99\% stenosis, or $100 \%$ occlusion). For patients who underwent preoperative carotid duplex ultrasound outside Cleveland Clinic $(1 \%)$ or in the radiology department at Cleveland Clinic (7\%), available study examination imaging or report of ultrasound velocity findings were used by a single investigator (H.K.) to grade the severity of carotid stenosis, using the standardized diagnostic criteria of the Cleveland Clinic Non-Invasive Vascular Laboratory.

\section{Endpoints}

Perioperative stroke was defined as any new focal or global neurologic deficit lasting $>24$ hours that could not be explained by another medical process. This included persistent neurologic deficit for $>72$ hours, and reversible ischemic neurologic deficit and recovery within 72 hours; excluded was transient ischemic attack with recovery within 24 hours. Temporal onset of stroke was classified as intraoperative if the neurologic deficit was present when the patient awoke from anesthesia, and postoperative if the deficit developed after the patient emerged from anesthesia.

\section{Statistical Analysis}

All analyses were performed using SAS, version 9.2 software (SAS Institute, Cary, NC). Continuous variables are summarized as mean $\pm \mathrm{SD}$, or as equivalent 15 th, 50 th (median), and 85 th percentiles when the distribution of values is skewed. Categoric data are summarized by frequencies and percentages.

\section{RESULTS}

\section{Screening Results}

Among the 1236 patients who had preoperative carotid ultrasound screening, $1069(86 \%)$ had no stenosis or mild stenosis, $90(7.3 \%)$ had moderate stenosis, and $77(6.2 \%)$ had severe stenosis (Figure 1). Patients with no stenosis or mild stenosis were younger than patients with moderate or severe stenosis. More of the patients with moderate or severe stenosis were women, and had heart failure, peripheral arterial disease, and a history of stroke and smoking (Table 1). Of those with moderate stenosis, most lesions were unilateral, equally distributed on the right $(50 \%)$ and left sides $(50 \%$; Table 2). Ten $(11 \%)$ of these patients had a history of stroke. Of those with severe stenosis, it was most commonly bilateral $(48 \%$; Table 3). Twelve $(16 \%)$ of these patients had a history of stroke.

\section{Management}

Of those with moderate carotid artery stenosis, $4(4.4 \%)$ had preoperative confirmatory testing (computed tomography angiography), and only $1(1.1 \%)$ underwent combined CABG and carotid endarterectomy (CABG + CEA). The latter patient had no history of stroke and was found to have severe carotid stenosis on confirmatory testing. Of those with severe stenosis, $18(23 \%)$ had confirmatory testing (computed tomography angiography $=16$, magnetic resonance angiography $=1$, angiogram $=1)$, and $18(23 \%)$ underwent $\mathrm{CABG}+\mathrm{CEA}$. Of the 18 undergoing CABG + CEA, 3 had a history of stroke; CABG + CEA was performed under the same anesthesia, with carotid 
TABLE 1. Patient characteristics stratified by degree of preoperative carotid stenosis

\begin{tabular}{|c|c|c|c|c|c|c|c|}
\hline \multirow[b]{2}{*}{ Characteristic } & \multicolumn{2}{|c|}{$\begin{array}{l}\text { No stenosis or mild stenosis } \\
\qquad(\mathrm{n}=1069)\end{array}$} & \multicolumn{2}{|c|}{$\begin{array}{l}\text { Moderate stenosis } \\
\quad(\mathbf{n}=\mathbf{9 0})\end{array}$} & \multicolumn{2}{|c|}{$\begin{array}{l}\text { Severe stenosis } \\
\quad(\mathbf{n}=77)\end{array}$} & \multirow[b]{2}{*}{$P$ value } \\
\hline & $\mathbf{n}^{*}$ & $\begin{array}{l}\text { No. }(\%) \text { or } \\
\text { mean } \pm \text { SD }\end{array}$ & $\mathbf{n}^{*}$ & $\begin{array}{l}\text { No. }(\%) \text { or } \\
\text { mean } \pm \text { SD }\end{array}$ & $\mathbf{n}^{*}$ & $\begin{array}{l}\text { No. }(\%) \text { or } \\
\text { mean } \pm \text { SD }\end{array}$ & \\
\hline \multicolumn{8}{|l|}{ Demographics } \\
\hline Age (y) & 1068 & $64 \pm 10$ & 90 & $69 \pm 9.9$ & 77 & $69 \pm 8.7$ & $<.0001$ \\
\hline Gender, female & 1068 & $240(22)$ & 90 & $37(41)$ & 77 & $30(39)$ & $<.0001$ \\
\hline Height $(\mathrm{cm})$ & 1068 & $173 \pm 9.6$ & 90 & $170 \pm 11$ & 77 & $170 \pm 9.8$ & .004 \\
\hline Body mass index $\left(\mathrm{kg} / \mathrm{m}^{2}\right)$ & 1068 & $30 \pm 5.8$ & 90 & $28 \pm 5.6$ & 77 & $29 \pm 5.6$ & .003 \\
\hline NYHA functional class & 841 & & 74 & & 68 & & .09 \\
\hline I & & $113(13)$ & & $9(12)$ & & $7(10)$ & \\
\hline II & & $375(45)$ & & $28(38)$ & & $22(32)$ & \\
\hline III & & $260(31)$ & & $28(38)$ & & $24(35)$ & \\
\hline IV & & $93(11)$ & & $9(12)$ & & $15(22)$ & \\
\hline \multicolumn{8}{|l|}{ Cardiac comorbidities } \\
\hline $\operatorname{LVEF}(\%)$ & 1049 & $51 \pm 11$ & 89 & $53 \pm 11$ & 77 & $50 \pm 12$ & .4 \\
\hline $\begin{array}{l}\text { No. of coronary artery systems diseased } \\
\quad(\geq 50 \% \text { stenosis })\end{array}$ & 1066 & & 90 & & 77 & & .16 \\
\hline 1 & & $62(5.8)$ & & $5(5.6)$ & & $0(0)$ & \\
\hline 2 & & 206 (19) & & $13(14)$ & & $13(17)$ & \\
\hline 3 & & $798(75)$ & & $72(80)$ & & $64(83)$ & \\
\hline Left main disease & 1065 & $283(27)$ & 90 & $27(30)$ & 77 & $28(36)$ & .15 \\
\hline Heart failure & 1068 & $277(26)$ & 90 & $29(32)$ & 77 & $32(42)$ & .007 \\
\hline \multicolumn{8}{|l|}{ Noncardiac comorbidities } \\
\hline Peripheral arterial disease & 1068 & $197(18)$ & 90 & $40(44)$ & 77 & $42(55)$ & $<.0001$ \\
\hline Prior stroke & 1068 & $81(7.6)$ & 90 & $10(11)$ & 77 & $12(16)$ & .03 \\
\hline Hypertension & 1068 & $998(93)$ & 90 & $86(96)$ & 77 & $73(95)$ & .7 \\
\hline Diabetes mellitus, pharmacologically treated & 1068 & $479(45)$ & 90 & $47(52)$ & 77 & $43(56)$ & .08 \\
\hline COPD & 1068 & $228(21)$ & 90 & $18(20)$ & 77 & $24(31)$ & .12 \\
\hline History of smoking & 1068 & $228(21)$ & 90 & $23(26)$ & 77 & $27(35)$ & .02 \\
\hline Creatinine (mg/dL) & 1062 & $0.97(0.77,1.3)$ & 90 & $1.0(0.71,1.5)$ & 77 & $1.1(0.79,1.8)$ & .003 \\
\hline Blood urea nitrogen (mg/dL) & 1062 & $20 \pm 8.8$ & 90 & $22 \pm 11$ & 77 & $23 \pm 10$ & .05 \\
\hline Bilirubin $(\mathrm{mg} / \mathrm{dL})$ & 1028 & $0.52 \pm 0.31$ & 89 & $0.45 \pm 0.26$ & 74 & $0.49 \pm 0.29$ & .05 \\
\hline Total cholesterol (mg/dL) & 939 & $159 \pm 46$ & 77 & $170 \pm 45$ & 66 & $162 \pm 54$ & .10 \\
\hline Triglycerides (mg/dL) & 939 & $144 \pm 110$ & 77 & $143 \pm 128$ & 66 & $136 \pm 69$ & .6 \\
\hline Hematocrit (\%) & 1066 & $40 \pm 5.6$ & 90 & $37 \pm 5.3$ & 77 & $37 \pm 6.3$ & $<.0001$ \\
\hline
\end{tabular}

Values are mean \pm SD, or No. (\%), unless otherwise indicated (creatinine is given as median [15th, 85th percentiles]). NYHA, New York Heart Association; $L V E F$, left ventricular ejection fraction; $C O P D$, chronic obstructive pulmonary disease. *Patients with data available.

endarterectomy performed first, and the wound left open. Afterward, coronary surgery was performed, followed by closure of chest and neck wounds. No patient in the study underwent carotid stenting or staged CEA followed by CABG. Off-pump surgery was performed in $75(7.0 \%)$ patients who had no stenosis or mild stenosis, $11(12 \%)$ who had moderate stenosis, and $6(7.8 \%)$ who had severe stenosis.

\section{Stroke}

By stenosis severity. Stroke occurred in 19 of $1382(1.4 \%)$ patients: 14 of $1069(1.3 \%)$ of those with no stenosis or mild carotid artery stenosis; 2 of $90(2.2 \%)$ of those with moderate stenosis; 2 of $77(2.6 \%)$ of those with severe stenosis $(P=.3)$; and 1 in a patient who was not screened. By management. In patients with moderate stenosis, 2 of $89(2.2 \%)$ strokes occurred in those undergoing isolated CABG, and no stroke occurred in the 1 patient who underwent $\mathrm{CABG}+\mathrm{CEA}$. For both patients who had a stroke, neither had had a prior stroke, and the stroke was bilateral hemispheric; 1 patient had been managed with cardiopulmonary bypass, and the other was an off-pump case.

In patients with severe stenosis, 1 of $59(1.7 \%)$ strokes that were ipsilateral to the carotid artery stenosis occurred in a patient undergoing isolated CABG; and 1 of 18 $(5.5 \%)$ strokes that were contralateral to the carotid stenosis occurred in patients undergoing CABG + CEA. Therefore, in patients with moderate or severe stenosis, 1 of $19(5.3 \%)$ undergoing $\mathrm{CABG}+\mathrm{CEA}$ and 3 of $148(2.0 \%)$ undergoing isolated CABG experienced stroke $(P=.4)$.

In patients who had no stenosis or mild stenosis, stroke occurred in 1 of $75(1.3 \%)$ undergoing off-pump surgery and 13 of $994(1.3 \%)$ undergoing on-pump surgery $(P=.9)$. In patients with moderate stenosis, stroke occurred 


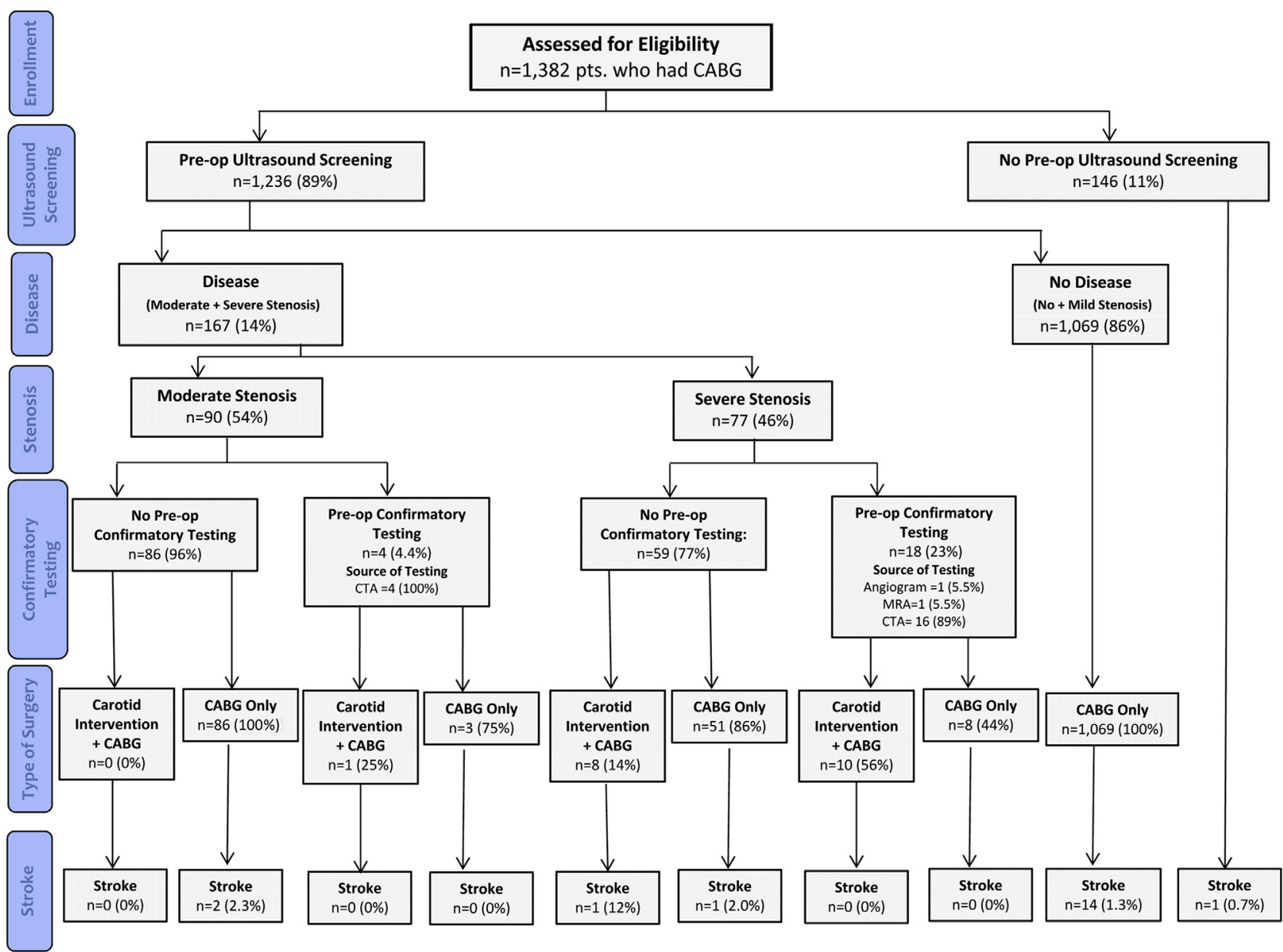

FIGURE 1. Diagram of study flow showing carotid artery ultrasound screening, its results, interventions, and endpoints. pts., Patients; $C A B G$, coronary artery bypass grafting; Pre-op, preoperative; CTA, computed tomography angiography; MRA, magnetic resonance angiography.

in 1 of $11(9.1 \%)$ undergoing off-pump surgery and 1 of $79(1.3 \%)$ undergoing on-pump surgery $(P=.2)$. In patients with severe stenosis, stroke occurred in 1 of 6 $(17 \%)$ undergoing off-pump surgery and 1 of $71(1.4 \%)$ undergoing on-pump surgery $(P=.15)$.

By timing and location. Of the 14 strokes that occurred in patients with no stenosis or mild stenosis, 3 were intraoperative, and 11 occurred postoperatively between days 1 and 13; stroke was bilateral in 8 patients (Table 4). Of the 2 strokes that occurred in patients who had moderate stenosis, 1 was intraoperative, and the other occurred

TABLE 2. Right and left coronary artery stenosis severity in patients with moderate stenosis

\begin{tabular}{lcc}
\hline Right stenosis (\% range) & Left stenosis (\% range) & No. $(\%)$ \\
\hline $0-19$ & $60-79$ & $4(4.4)$ \\
$20-39$ & $60-79$ & $25(28)$ \\
$40-59$ & $60-79$ & $16(18)$ \\
$60-79$ & $0-19$ & $2(2.2)$ \\
$60-79$ & $20-39$ & $16(18)$ \\
$60-79$ & $40-59$ & $27(30)$ \\
\hline
\end{tabular}

postoperatively, on day 3; stroke was bilateral hemispheric in both. Of the 2 strokes that occurred in patients who had severe stenosis, 1 occurred on postoperative day 2 and the other on postoperative day 4; stroke was unilateral in both.

\section{DISCUSSION \\ Principal Findings}

Routine, nonselective carotid artery ultrasound screening in our institution altered the management of only a minority of the patients who were found to have moderate or severe carotid artery stenosis. The minority of these went on to confirmatory testing and carotid revascularization. Patients with moderate or severe carotid artery disease did, in fact, experience more perioperative strokes. However, CEA did not seem to prevent these. The choice of performing CABG either on- or off-pump did not seem to be influenced by the severity of carotid artery stenosis, and the occurrence of stroke was similar in patients undergoing either type of surgery, with mild, moderate, or severe stenosis. The 
TABLE 3. Right and left coronary artery stenosis severity in patients with severe stenosis

\begin{tabular}{lc}
\hline \multicolumn{1}{c}{ Stenosis type } & No. $(\%)$ \\
\hline Bilateral, $60 \%-79 \%$ & $36(47)$ \\
Occluded unilateral & $20(26)$ \\
Unilateral, $80 \%-99 \%$ & $15(19)$ \\
ICA occlusion and contralateral, $80 \%-99 \%$ & $3(3.9)$ \\
CCA occluded & $1(1.3)$ \\
Bilateral, $80 \%-99 \%$ & $1(1.3)$ \\
CCA occlusion and contralateral, $80 \%-99 \%$ & $1(1.3)$ \\
\hline
\end{tabular}

ICA, Internal carotid artery; $C C A$, common carotid artery.

laterality of the stroke in the 3 patients who had moderate or severe stenosis and underwent CABG alone was ipsilateral to the carotid stenosis in only 1 patient, making the cause of perioperative stroke in the other 2 patients unlikely to be related to carotid disease.

\section{Findings in Context}

Other studies have reported findings similar to ours. Fukuda and colleagues ${ }^{3}$ found that nonselective carotid artery ultrasound screening resulted in carotid revascularization in only 11 of $308(4 \%)$ patients. In their study, 2 strokes occurred: 1 in a patient with $>50 \%$ stenosis who underwent combined CABG + CEA, and 1 in a patient who had no stenosis or mild stenosis. No strokes occurred in the 33 patients with $>50 \%$ stenosis who did not undergo carotid revascularization. Salehiomran and colleagues ${ }^{2}$ performed routine screening of 1604 patients that led to carotid revascularization in only 5 . In their study, no patients who had significant carotid stenosis $(>60 \%)$ had a stroke, whereas 22 strokes occurred in patients who did not have significant carotid stenosis. In addition, studies have shown that the risk of stroke in patients with carotid disease is low, ${ }^{9-11}$ and carotid stenosis has no direct causal relationship with ipsilateral stroke. ${ }^{10}$

Because patients with significant carotid stenosis are likely to have an atherosclerotic aorta, off-pump surgery should be considered, to avoid aortic manipulation. However, in our study, carotid stenosis did not influence the surgeon's choice of performing CABG on-pump versus off-pump, and off-pump surgery did not reduce stroke risk.

Several other strategies can be considered as a means to reduce the occurrence of stroke in patients undergoing CABG, including intraoperative epiaortic scanning and monitoring of blood pressure throughout the procedure. We recommend performing intraoperative epiaortic scanning in all patients undergoing CABG to identify those in whom aortic manipulation should be avoided. In patients with severe carotid stenosis, avoiding extremes of blood pressure is important, because hypotension can cause cerebral ischemia, and hypertension can cause plaque rupture. Off-pump surgery can be considered for patients who have severe carotid stenosis, to avoid hypotension, which may occur during use of cardiopulmonary bypass. Present-day carotid screening focuses on decreasing the occurrence of intraoperative stroke via use of these techniques; however, it does not appear to do so. We know from this study and our previous one ${ }^{1}$ that most strokes occur postoperatively, not intraoperatively. Therefore, postoperative antiplatelet therapy should be

TABLE 4. Perioperative stroke location and carotid artery disease

\begin{tabular}{|c|c|c|c|c|c|}
\hline Carotid stenosis & Right (\% range) & Left ( $\%$ range $)$ & No. of patients & Stroke location & Postoperative day \\
\hline No screening & - & - & 1 & Left & 6 \\
\hline \multicolumn{6}{|c|}{ No stenosis or mild stenosis } \\
\hline & $0-19$ & $0-19$ & 1 & Bilateral & 1 \\
\hline & $20-39$ & $20-39$ & 7 & Bilateral & 1 \\
\hline & & & & Bilateral & 13 \\
\hline & & & & Right & 6 \\
\hline & & & & Left & 1 \\
\hline & & & & Left & 2 \\
\hline & & & & Left & 5 \\
\hline & & & & Left & 11 \\
\hline & $20-39$ & $40-59$ & 3 & Bilateral & Upon awakening \\
\hline & & & & Bilateral & 3 \\
\hline & & & & Right & Upon awakening \\
\hline & $40-59$ & $20-39$ & 2 & Bilateral & Upon awakening \\
\hline & & & & Bilateral & 3 \\
\hline & $40-59$ & $40-59$ & 1 & Bilateral & 4 \\
\hline \multicolumn{6}{|l|}{ Moderate stenosis } \\
\hline & $60-79$ & $40-59$ & 2 & Bilateral & Upon awakening \\
\hline & & & & Bilateral & 3 \\
\hline \multicolumn{6}{|l|}{ Severe stenosis } \\
\hline & 20-39 & $80-99$ & 1 & Right & 4 \\
\hline & 100 (occluded) & $20-39$ & 1 & Right & 2 \\
\hline
\end{tabular}


considered for patients undergoing CABG, because most postoperative strokes result from thromboembolic events.

\section{Routine Versus Selective Screening}

Based on the results of this study, we have changed our approach from use of routine screening to use of selective screening for carotid stenosis in CABG patients. We now screen only those patients who have a history of stroke or of transient ischemic attack, bruit, left main disease, previous CEA or stenting, amaurosis fugax, or new-onset extremity weakness.

Studies have shown that selective screening in certain high-risk patients can detect $\geq 50 \%$ carotid stenosis with $100 \%$ sensitivity. ${ }^{12}$ These high-risk patients include women and those with a history of diabetes, hypertension, peripheral arterial disease, carotid disease, stroke, left main coronary disease, and smoking. Durand and colleagues $^{5}$ conducted a study to determine which of 2 approaches-selective or nonselective preoperative carotid screening - is most clinically efficacious. They found that selective screening using a risk-profiling algorithm based on patients being aged $>65$ years, having carotid bruit on exam, and having a history of stroke would have reduced the screening load by $40 \%$, with a negligible effect on either surgical management or stroke. Given these findings and the findings of our study, a more targeted approach for preoperative carotid artery evaluation seems reasonable. Additionally, guidelines recommend screening in selected patients only. ${ }^{9,13-17}$

\section{Limitations}

The association of surgical intervention with stroke risk was determined observationally and may be confounded by unmeasured covariables. In addition, because the occurrence of stroke was low, the study was underpowered to detect significant differences. Further, carotid revascularization can be performed using staged or synchronous CEA or carotid artery stenting; however, patients in this study underwent only synchronous CABG and CEA. No one underwent carotid stenting or staged CEA followed by CABG. Because individual surgeons have interpreted the available data differently, some have performed combined CABG + CEA for important carotid stenosis, and others have not. In general, the rationale for making either decision was not documented in the medical record.

Classification of the timing of stroke occurrence is somewhat arbitrary and sometimes challenging in the setting of anesthesia use, as well as postoperative delirium and other complications. Additionally, we were unable to determine whether each stroke was embolic, thrombotic, or hypoperfusive in etiology. Finally, these results are from a single academic medical center and may not be generalizable.

\section{CONCLUSIONS}

Nonselective preoperative carotid artery screening altered the management of a minority of patients undergoing $\mathrm{CABG}$. These changes did not translate into a decrease in perioperative stroke risk. Given this observation, carotid artery disease might be only a surrogate marker of patients' diffuse atherosclerotic burden. The most compelling evidence from this study emerges from the stroke patterns observed-many more postoperative than intraoperative events, approximately equal numbers of bilateral versus unilateral events, and an absence of correlation between unilateral events and laterality of carotid disease when it was present. This information strongly suggests that the incremental benefit of carotid screening, even if present, is likely to be small. Hence, in patients undergoing $\mathrm{CABG}$, a more targeted approach for preoperative carotid artery evaluation should be adopted.

\section{Conflict of Interest Statement}

Authors have nothing to disclose with regard to commercial support.

You can watch a Webcast of this AATS meeting presentation by going to: http://webcast.aats.org/2015/ Video/Tuesday/04-28-15_4E_1615_Masabni.mp4.

\section{References}

1. Tarakji KG, Sabik JF III, Bhudia SK, Batizy LH, Blackstone EH. Temporal onset, risk factors, and outcomes associated with stroke after coronary artery bypass grafting. JAMA. 2011;305:381-90.

2. Salehiomran A, Shirani S, Karimi A, Ahmadi H, Marzban M, Movahedi N, et al. Screening of carotid artery stenosis in coronary artery bypass grafting patients. J Tehran Heart Cent. 2010;5:25-8.

3. Fukuda I, Gomi S, Watanabe K, Seita J. Carotid and aortic screening for coronary artery bypass grafting. Ann Thorac Surg. 2000;70:2034-9.

4. Anastasiadis K, Karamitsos TD, Velissaris I, Makrygiannakis K, Kiskinis D. Preoperative screening and management of carotid artery disease in patients undergoing cardiac surgery. Perfusion. 2009;24:257-62.

5. Durand DJ, Perler BA, Roseborough GS, Grega MA, Borowicz LM Jr, Baumgartner WA, et al. Mandatory versus selective preoperative carotid screening: a retrospective analysis. Ann Thorac Surg. 2004;78:159-66; discussion 166.

6. Shirani S, Boroumand MA, Abbasi SH, Maghsoodi N, Shakiba M, Karimi A et al. Preoperative carotid artery screening in patients undergoing coronary artery bypass graft surgery. Arch Med Res. 2006;37:987-90.

7. Lee EJ, Choi KH, Ryu JS, Jeon SB, Lee SW, Park SW, et al. Stroke risk after coronary artery bypass graft surgery and extent of cerebral artery atherosclerosis. J Am Coll Cardiol. 2011;57:1811-8.

8. Olin JW, Fonseca C, Childs MB, Piedmonte MR, Hertzer NR, Young JR. The natural history of asymptomatic moderate internal carotid artery stenosis by duplex ultrasound. Vasc Med. 1998;3:101-8.

9. Masabni K, Raza S, Blackstone EH, Gornik HL, Sabik JF. Does preoperative carotid stenosis screening reduce perioperative stroke in patients undergoing coronary artery bypass grafting? J Thorac Cardiovasc Surg. 2015;149: 1253-60.

10. Li Y, Walicki D, Mathiesen C, Jenny D, Li Q, Isayev Y, et al. Strokes after cardiac surgery and relationship to carotid stenosis. Arch Neurol. 2009;66:1091-6.

11. Ghosh J, Murray D, Khwaja N, Murphy MO, Walker MG. The influence of asymptomatic significant carotid disease on mortality and morbidity in patients undergoing coronary artery bypass surgery. Eur J Vasc Endovasc Surg. 2005; 29:88-90. 
12. Sheiman RG, Janne d'Othee B. Screening carotid sonography before elective coronary artery bypass graft surgery: Who needs it. AJR Am J Roentgenol. 2007; 188:W475-9.

13. Hillis LD, Smith PK, Anderson JL, Bittl JA, Bridges CR, Byrne JG, et al. 2011 ACCF/AHA Guideline for Coronary Artery Bypass Graft Surgery. A report of the American College of Cardiology Foundation/American Heart Association Task Force on Practice Guidelines. Developed in collaboration with The American Association for Thoracic Surgery, Society of Cardiovascular Anesthesiologists, and Society of Thoracic Surgeons. Executive summary. J Am Coll Cardiol. 2011:58:2584-614.

14. The Society of Thoracic Surgeons. Five things physicians and patients should question. Choosing Wisely. An initiative of the ABIM Foundation. Available at: http://www.choosingwisely.org/doctor-patient-lists/the-society-of-thoracicsurgeons/. Accessed September 4, 2013.

15. Brott TG, Halperin JL, Abbara S, Bacharach JM, Barr JD, Bush RL, et al. 2011 ASA/ACCF/AHA/AANN/AANS/ACR/ASNR/CNS/SAIP/SCAI/SIR/SNIS/SVM/ SVS guideline on the management of patients with extracranial carotid and vertebral artery disease: executive summary: a report of the American College of Cardiology Foundation/American Heart Association Task Force on Practice Guidelines, and the American Stroke Association, American Association of Neuroscience Nurses, American Association of Neurological Surgeons, American College of Radiology, American Society of Neuroradiology, Congress of Neurological Surgeons, Society of Atherosclerosis Imaging and Prevention, Society for Cardiovascular Angiography and Interventions, Society of Interventional Radiology, Society of NeuroInterventional Surgery, Society for Vascular Medicine, and Society for Vascular Surgery. J Am Coll Cardiol. 2011;57:1002-44.

16. Mohler ER III, Gornik HL, Gerhard-Herman M, Misra S, Olin JW, Zierler RE, et al. ACCF/ACR/AIUM/ASE/ASN/ICAVL/SCAI/SCCT/SIR/SVM/SVS/SVU [corrected] 2012 appropriate use criteria for peripheral vascular ultrasound and physiological testing part I: arterial ultrasound and physiological testing: a report of the American College of Cardiology Foundation appropriate use criteria task force, American College of Radiology, American Institute of Ultrasound in Medicine, American Society of Echocardiography, American Society of Nephrology, Intersocietal Commission for the Accreditation of Vascular Laboratories, Society for Cardiovascular Angiography and Interventions, Society of Cardiovascular Computed Tomography, Society for Interventional Radiology, Society for Vascular Medicine, Society for Vascular Surgery, [corrected] and Society for Vascular Ultrasound. [corrected]. J Am Coll Cardiol. 2012;60:242-76.

17. Qureshi AI, Alexandrov AV, Tegeler CH, Hobson RW II, Dennis Baker J, Hopkins LN. Guidelines for screening of extracranial carotid artery disease: a statement for healthcare professionals from the multidisciplinary practice guidelines committee of the American Society of Neuroimaging; cosponsored by the Society of Vascular and Interventional Neurology. J Neuroimag. 2007; $17: 19-47$.

Key Words: carotid stenosis, carotid endarterectomy, ultrasound screening, coronary artery bypass grafting

\section{Discussion}

Dr T. E. MacGillivray (Boston, Mass). Thank you very much, Dr Pomar, Dr Sundt. I am grateful to Dr Masabni for sending me a copy of his manuscript in advance, and I congratulate you on an excellent presentation. Back when I was a cardiac surgery resident, one of my attendings would frequently lament, "If it weren't for the bleeding and the strokes, cardiac surgery would be the greatest job in the world!" Although we all know that bleeding eventually stops, the catastrophic impact of stroke can last forever. In cardiac surgery patients, stroke continues to be a leading cause of perioperative morbidity and mortality.

In patients undergoing $\mathrm{CABG}$, the risk factors for coronary artery disease are the risk factors for cerebrovascular disease and stroke as well. It has been well established that CABG patients with significant carotid stenosis are at increased risk of stroke. According to The Society of Thoracic Surgeons database, the risk of stroke during $\mathrm{CABG}$ is $2 \%$ when the patient has severe stenosis, defined as greater than $75 \%$ carotid artery disease, compared to a $1 \%$ risk of stroke in patients without significant carotid disease. These may seem like small numbers, but another way to look at it is that, with severe carotid stenosis, the risk of stroke is doubled in our CABG patients.

Logically, it would follow that, by casting a broad net with a liberal use of carotid ultrasound screening, we may catch severe carotid stenosis, allowing us to change our operative plan, and subsequently, the outcome. Unfortunately, multiple reports have demonstrated that unilateral carotid stenosis is not only a risk of stroke, but also a marker that the risk of stroke is increased, not only in the ipsilateral case, but also, as we saw today, in the contralateral sides as well. In addition, there continues to be debate as to whether carotid intervention with CABG improves neurologic outcome.

This report from the Cleveland Clinic experience with nonselective carotid artery ultrasound screening in patients undergoing coronary artery bypass shows that, in a period of 2-1/2 years, almost $90 \%$ of their isolated CABG patients had carotid duplex studies. Even though they identified severe carotid artery disease in $6.2 \%$ of those patients, their conclusions were that routine carotid evaluation altered the management in only a minority of these patients, and that did not seem to translate into a decreased risk in the perioperative stroke rate. On that basis, they are advocating for a more targeted approach for the use of preoperative carotid screening, which is certainly a positive movement toward being good stewards of health care resources.

I have 3 questions. Although the Cleveland Clinic is world renowned for large volumes of coronary surgery, the cohort of patients that had a high risk of stroke from carotid disease was actually quite small; it was 77 patients. Given that the expected risk of perioperative stroke in those high-risk patients was only approximately $2 \%$, do you think that your study was sufficiently powered to be statistically meaningful? I guess the question is: If you had more patients, do you think the results would be statistically significant?

Dr K. Masabni (Cleveland, Ohio). Thank you for this question. Basically, you are correct. There were only 77 patients who had severe carotid artery stenosis, and only 2 of those 77 developed stroke; interestingly, 1 of those 2 patients underwent combined CABG and carotid revascularization. However, the main purpose of our study was to look at whether routine screening is beneficial, and of the 1236 patients who were screened in our patient population, only 77 were found to have severe stenosis, 
and only 2 developed stroke. So the value of routine screening is questionable, and I think it is not valuable.

Dr MacGillivray. In your conclusion, you state that management was altered in only the minority of patients. Of the 77 patients with severe carotid stenosis, only $23 \%$ had a carotid intervention. Although $7 \%$ of the patients had off-pump CABG, it seemed as if those patients were evenly distributed across the spectrum of patients with carotid stenosis.

Could you please clarify how it was decided which patients received a CEA with their CABG, and how the patients were chosen to receive off-pump rather than on-pump CABG? Do you have established treatment protocols, or is it surgeon dependent, and do you think that it might possibly have affected your results?

Dr Masabni. You are correct. The choice of performing coronary surgery on pump or off pump did not seem to be influenced by the severity of carotid disease. In fact, the occurrence of stroke in patients with mild, moderate, or severe carotid stenosis was not influenced by whether the operations were performed on pump or off pump, and the approach chosen was completely surgeon dependent. At Cleveland Clinic, there are no current protocols for whether patients undergo combined coronary surgery and CEA. However, it has been obvious that most patients who have bilateral severe carotid disease and those who have occluded unilateral carotid disease and a contralateral moderate or severe stenosis tend to undergo CEA combined with their coronary surgery.

Dr MacGillivray. For my last question-could you please tell us how the Cleveland Clinic has changed its practice, and how does the Cleveland Clinic now select which patients get carotid ultrasound evaluation?

Dr Masabni. Currently, only patients who are found to have a history of stroke or transient ischemic attack, those who have a carotid bruit, and those who have peripheral vascular disease, in addition to those with high-risk disease such as left main, are screened preoperatively before their coronary revascularization.

Dr T. Sundt (Boston, Mass). If you look at the overall practice, what was the stroke rate in the 2 years before you instituted this practice, and the stroke rate overall during this study?

Dr Masabni. According to prior studies done at Cleveland Clinic, the occurrence of stroke has been low, around $1 \%$ to $2 \%$. As we can see, stroke occurrence now has ranged from $1.3 \%$, for patients with no stenosis, up to $2.6 \%$ in those with severe stenosis.

Dr Sundt. But if you look at the practice during the 2 years before this-just those 2 years-do you know what the stroke rate was in those 2 years?

Dr Masabni. It was between $1 \%$ and $2 \%$.

Dr G. H. Almassi (Milwaukee, Wis). I believe the CARP (Coronary Artery Revascularization Prophylaxis) study, more than 10 years ago, looking at the same issue, showed that in patients who were symptomatic, regardless of the degree of stenosis, you need to treat that symptomatic patient-ie, if the patient has carotid artery symptoms, then regardless of the extent of the coronary artery disease, you take care of the carotid; and vice versa-if the patient has coronary disease, and at the same time carotid disease, you leave it alone. That was the CARP study.

My question to you is: What was the reason for these patients having carotid screening? You did not do it for all your patients. Did they have symptoms, or was there a score in terms of their extent of atherosclerosis that made you do screening?

Dr Masabni. As I mentioned earlier, this was a quality improvement protocol in which we planned on screening every patient who had planned isolated CABG, and per the results, $89 \%$ of the entire patient population who had surgery at Cleveland Clinic were screened; only $11 \%$ of the patients who had CABG were not screened, basically only because of emergency medical circumstances.

Dr Almassi. So none of the patients who had carotid stenosis had symptoms related to carotid disease?

Dr Masabni. Some of the patients had symptoms of carotid disease, and they were part of the group who were screened.

Dr Sundt. Do you routinely do epicardial aortic ultrasound?

Dr Masabni. This is actually surgeon dependent, but we do screen patients for atherosclerotic disease intraoperatively, using either palpation of the aorta or epiaortic ultrasound screening. We recommend that surgeons do epiaortic ultrasound screening, because this could decrease the risk of intraoperative and postoperative stroke. In fact, we think that because most strokes occur postoperatively late or after surgery, the cause of postoperative stroke is actually thromboembolic in nature, and that could be a result of debris from the aorta shedding to the carotid cerebral arteries and causing stroke. So postoperative antiplatelet therapy is 1 of the things we should probably consider for patients undergoing coronary surgery.

Dr J.-L. Pomar (Barcelona, Spain). May I ask you whether you routinely do an ultrasound on those patients or not?

Dr MacGillivray. Routinely do what?

Dr Pomar. An ultrasound for carotid stenosis in the CABG patients at your institution.

Dr MacGillivray. For the past several years, we have had a targeted approach, to use the Cleveland Clinic's words. We have a set of risk factors, including age, risk of previous stroke, and the presence of carotid bruits. But we have a targeted approach. We do not do that [ultrasound] nonselectively. 